\title{
Consideraciones en torno a la globalización en el marco de una hermenéutica ricoeuriana
}

\author{
Esteban Lythgoe *
}

\begin{abstract}
Resumen: En la presente se propone a la hermenéutica de Ricoeur como una alternativa para resolver las tensiones entre los procesos de globalización desde abajo y globalización desde arriba. Si bien el espíritu de esta propuesta coincide con la de Kristensson Uggla, rechazamos su tesis de que el filósofo francés no aborda la temática de la globalización. Evaluamos la propuesta de Ricoeur sobre este punto, y luego señalamos el modo en que su consideración afecta a la aplicación de la hermenéutica en problemas concretos. Se reconoce finalmente la importancia que ha tenido la recepción del giro lingüístico en historia para la aplicación de la hermenéutica en problemáticas sociales.
\end{abstract} dón.

Palabras clave: Globalización - traducción - intercambio de memoria - per-

Abstract: In this paper Ricoeur's hermeneutics is proposed as an alternative for solving the tensions of the processes of "globalization from above" and "globalization from below". Although the spirit of our proposal is similar to Kristensson Uggla's, we reject his theses that Ricoeur didn't develop anything about globalization. We evaluate the philosopher's proposal on this issue and show how its consideration would have altered Uggla's application of hermeneutics in concrete situations. Finally, we recognize the importance of the linguistic turn in history for the application of hermeneutics in social problems.

Key words: Globalization - translation - Exchange of memory - forgiveness.

* Doutor em filosofía pela Universidade de Buenos Aires (UBA).

Latitude, Vol. 4, n², pp. 115-126, 2010

DOI: https://doi.org/10.28998/2179-5428.20100207 
Consideraciones en torno a la globalización en el marco de una hermenéutica rico euriana

La globalización se ha convertido en una de las temáticas que más debates ha suscitado en estos últimos años, tanto en el ámbito político como en el académico. Existe una gran coincidencia respecto a sus manifestaciones. Así, se la ha caracterizado como un proceso de integración cultural y económica global debida fundamentalmente al desarrollo de las nuevas tecnologías de información y de logística, que están produciendo un dominio a nivel global del sistema económico capitalista. Este proceso supone conflictos y contradicciones que han sido puestos de manifiesto por varios autores, ${ }^{1}$ pues, por una parte, horadando las culturas y tradiciones locales a favor de una cultura global, pero, por la otra, proporciona a sectores marginados el acceso a información, herramientas e instituciones que hasta hace un tiempo les resultaba inaccesible.

Uno de los puntos más urticantes es la tensión entre dos proceso integradores diferentes, uno desde abajo y otro desde arriba. "La 'globalización desde abajo' se refiere a los modos en que individuos marginalizados y movimientos sociales resisten a la globalización y/o utilizan sus instituciones e instrumentos para una mayor democratización y justicia social. Mientras en un nivel la globalización aumenta significativamente la supremacía de las grandes corporaciones y gobiernos, también da poder a grupos e individuos que previamente eran dejados fuera del diálogo democrático y del terreno de la lucha política." (Kellner, 2010, p. 193). Si bien este concepto ha sido acuñado hace más de dos décadas por teóricos sociales y se pueden encontrar antecedentes históricos de la temática que datan del siglo XIX, ${ }^{2}$ aún no habido importantes logros desde el punto de vista de los análisis teóricos. Este hecho ha reforzado la indagación teórica, en lugar de frustrarla, dando lugar a obras, a primera vista, insospechados. Este es el caso de la hermenéutica, y específicamente de Paul Ricoeur, cuyos últimos desarrollos aportan, en mi opinión, algunos elementos para reencauzar la tensión entre estos dos modos de concebir el proceso de globalización. En este sentido, retomo la propuesta presentada por Bengt Kristensson Uggla, en su obra de reciente publicación Ricoeur, Hermeneutics and Globalization. En ella se sostiene que los aportes de Ricoeur a la teoría de la interpretación, específicamente, la instancia de la distanciación y la aspiración de llevar a cabo mejores interpretaciones, han influido positivamente en el desa-

${ }^{1}$ Cf. entre otros Kellner, 2002; Hardt y Negri, 2000; Friedman, 1999; Stiglitz, 2002.

${ }^{2}$ Cf. http://plato.stanford.edu/entries/globalization/ 
rrollo de la hermenéutica, constituyéndola en un abordaje clave para dar cuenta de este proceso, a punto tal que califica a esta época la era de la hermenéutica.

El presente artículo consta de tres partes. La primera es una respuesta a una de las tesis de este autor, según la cual, "a pesar de que Ricoeur mismo nunca entró en reflexiones series sobre la globalización, con su profunda transformación del concepto de interpretación ha empero abierto la puerta para que se puedan dar los pasos posteriores." (Uggla, 2010, p. 52). Más que utilizar las herramientas que proporciona la hermenéutica de Ricoeur para teorizar acerca de la globalización, el autor recurre a ella como vía para resolver algunos de los dilemas a los que nos enfrenta este proceso. Así, apoyándose fundamentalmente en las caracterizaciones de la actualidad realizadas por G. Vattimo, según la cual no hay hechos estables, sino interpretaciones variables, la hermenéutica es presentada como una estrategia de racionalidad alternativa al relativismo horizontal postmoderno y al intento del pensamiento vertical de jerarquías fijas de verdad. ${ }^{3}$ Frente a esta tesis traeremos brevemente a colación las tesis centrales del artículo de Ricoeur, "Reflections on a new ethos for Europe", donde se proponen "las condiciones éticas y políticas de posibilidad" de la integración de bloques supranacionales. En segundo lugar, quisiéramos poner de manifiesto las limitaciones que tiene de la visión de Uggla respecto de la de Ricoeur. Para ello, nos remitiremos a la aplicación que hace este intérprete de las categorías hermenéuticas al caso de la identidad colectiva nórdica. Allí se pondrá de manifiesto una suerte de terapéutica hermenéutica, no mencionada por este intérprete, pero que considero de utilidad para las tensiones ligadas a la globalización. Quisiera finalmente evaluar algunos motivos por los que la hermenéutica se ha convertido en una de las corrientes que busca resolver algunas de las tensiones ligadas con la globalización. En efecto, la globalización, tanto sea historia global como la discusión en torno a procesos de integraciones regionales, es una problemática asociada con la teoría e historia social. La hermenéutica, por su parte, está ligada fundamentalmente con teoría del texto que ha influido fuertemente en el desarrollo de la historia intelectual. La pregunta es, pues, cómo es posible pueda desplazarse hacia la teoría social y sostenga que "...la historia social no es un sector entre otros, sino el punto de vista bajo el cual la historia elige su campo, el de las ciencias sociales." (Ricoeur, 2000, 232). La pregunta es, pues, ¿qué ha permitido en esta obra este desplazamiento de cuestiones ligadas con la interpretación de textos a problemáticas sociales?

${ }^{3}$ Cf. Uggla, 2010, p. 61. 
Consideraciones en torno a la globalización en el marco de una hermenéutica rico euriana

Quisiéramos esbozar brevemente las principales tesis de un artículo acerca del proceso de integración europeo, elaborado por Ricoeur, en la década de los noventa, como respuesta a la tesis de Uggla de que Ricoeur nunca ha teorizado acerca de la globalización. En este artículo, el filósofo francés elabora un modelo de tres etapas para la integración de lo mismo y lo otro como condición de posibilidad de la constitución de bloques supranacionales. Entre estas etapas existe una relación de fundamentación y de estrechez de vínculos. Esto significa que cada nivel de integración es la condición de posibilidad para el siguiente y que, cuantos más niveles se compartan, más estrecho será el vínculo entre ambos. Asimismo, veremos que, desde el punto de vista interno, es posible establecer una suerte de nexo genético entre cada una de estas etapas y los desarrollos de Ricoeur a partir de la década de los setenta.

El primer nivel hace alusión a la cuestión de la traducción entre idiomas. El primer punto de interés es que el filósofo no concibe a la multiplicidad de idiomas como un obstáculo en el proceso integrador, sino que considera a la pluralidad lingüística como un atributo constitutivo del ser humano. Es por este motivo que hay una oposición respecto de todos aquellos proyectos modernos de superar el mito de Babel a través de la imposición de una lengua natural o la creación de una lengua artificial, y una insistencia en la necesidad de que cualquier bloque multilingüístico permanezca sea políglota. En segundo lugar, no se interna en el problema empírico sobre la posibilidad de la traducción. En su lugar, postula a la necesidad de establecer a la traductibilidad como un a priori de la comunicación. Así explica, "voy a hablar de 'principio universal de traductibilidad'. La traducción es de facto; la traductibilidad es de jure." (Ricoeur, 1995, p. 4). Este principio establece la posibilidad de trasladar de un idioma a otro aquello que se intenta comunicar, sea de manera oral como escrita, sin que haya una pérdida irreparable en su contenido semántico. El proceso de traducción consiste en establecer la relación óptima entre los recursos de la lengua que se recibe y la que se emite. Aquí se presenta, por primera vez, el dilema entre lo mismo y lo otro, pues el traductor debe determinar si privilegiar los recursos de la lengua propia, siguiendo el modelo integrador propuesto por San Agustín, o buscar adecuarse a los recursos de la lengua del otro, tal como fue propuesto por el modelo de hospitalidad de von Humbolt. Ricoeur se inclina por el segundo, considerando que existe una asimetría a favor del traductor que debe ser enmendada. Quien es traducido tiene limitada su capacidad de poder-decir; corresponde, pues, al traductor romper con esta asimetría. Además de instarnos al aprendizaje de más de un idioma, este dilema y su resolución son utilizados por Ricoeur como paradigma para los demás niveles de análisis, quien nos exhorta a extender lo que el filósofo denomina ethos de la traducción de lo meramente lingüístico a lo cultural y espiritual.

Mientras el estrato anterior se encuentra fuertemente ligado a los análisis más semiológicos que realizara el filósofo durante las década de los setenta y comienzos de los ochenta, el siguiente nivel, asociado con el "intercambio de memorias", toma como 
punto de partida sus consideraciones en torno a la identidad narrativa de la década de los noventa y sus análisis acerca del reconocimiento y la memoria, de los años dos mil.

Con respecto a la memoria, pese a que su herencia fenomenológica lo lleva a compartir varios elementos de la denominada tradición de la mirada interior, es decir, de autores como Agustín, Locke y Kant, Ricoeur es consciente que ella es incapaz de dar cuenta de toda una serie de fenómenos sociales que han logrado ser explicados por la línea sociológica de la memoria colectiva iniciada por M. Halbwachs. Su propuesta conceptual, entonces, es proponer una definición de memoria capaz de proporcionar un marco conceptual de diálogo entre ambas tradiciones.

En este sentido, resultan fundamentales los aportes de H. Bergson y P. F. Strawson. La primera dificultad para una definición hermenéutica de la memoria surge de la pregunta fundamental acerca de dónde se almacena la memoria y su tradicional respuesta que remite la memoria al cerebro. En primer lugar, Bergson le quitó importancia a esta pregunta reemplazándola por la de cómo se recuerda. En segundo lugar, desvinculó a la memoria del cerebro, tomando como punto de partida su oposición entre representación y acción: la memoria quedó asociada con la representación y contrapuesta a la corporalidad en tanto órgano de acción cuyo centro organizador es el cerebro. ${ }^{4}$

Los planteos de Strawson sobre la atribución múltiple, por su parte, le proporcionan a Ricoeur los elementos necesarios para sostener una región lingüística en la que concuerden los discursos mnémicos de la tradición sociológica y de la mirada interior. ${ }^{5}$ Según la tesis desarrollada en Los individuos, para encuadrarse dentro de este ámbito lingüístico, los predicados deben cumplir las siguientes condiciones: 1 . la atribución debe poder ser suspendida u operada, 2. los predicados deben guardar el mismo sentido en dos situaciones de atribución diferentes, 3. esta atribución múltiple debe mantener la disimetría entre adscripción a sí mismo y adscripción al otro. La primera parte de La memoria, la historia el olvido es una muestra del cumplimiento de la primer condición, pues en ella se desimplica al qué y al cómo de la memoria de quién recuerda. Con respecto a la segunda condición, más allá del estrecho vínculo entre el recuerdo y quien recuerda, la suspensión de la atribución permite adscribir la memoria a otro, sin por ello modificar su sentido. A pesar de ello, la condición de la disimetría también se cumple, pues, pese a poder trasladarse la atribución de un recuerdo, uno es incapaz de plenificar o confirmar dicha atribución.

Tomando los elementos conceptuales recién señalados el filósofo proporciona una definición hermenéutica de la memoria de la siguiente manera: "pero hablar de memoria no es sólo evocar una facultad psico-fisiológica que está asociada con la pre-

${ }^{4}$ Cf. Ricoeur, 2000, 554 y ss.
${ }^{5}$ Cf. Ricoeur, 2000, 152-157.

Latitude, Vol. 4, n² 2, pp. 115-126, 2010. 
Consideraciones en torno a la globalización en el marco de una hermenéutica rico euriana

servación y recolección de trazos del pasado; es presentar la función 'narrativa' a través de la cual esta capacidad primaria de preservación y recolección es ejercida en el nivel público del lenguaje." (Ricoeur, 1995, p. 6).

La identidad narrativa, por su parte, se plantea como la alternativa entre una propuesta sustancialista al problema de la identidad personal y el escepticismo en torno a este tema fundado fundamentalmente en los ataques de autores como Hume y Nietzsche. Según la hermenéutica, la identidad sería un constructo narrativo, donde los acontecimientos vividos se constituyen en una totalidad por medio de la narración de una vida. En efecto, "al mismo tiempo que las acciones relatadas reciben la unidad temporal de una historia de la trama, los caracteres de la historia pueden también ser considerados puestos en intriga (mise en intrigue) (Ricoeur, 1995, p. 6). Hay tres elementos de la definición hermenéutica de la identidad que la vuelven tan importante para el actual nivel de análisis. En primer lugar, la identidad deja de ser considerada como un hecho inconmovible y se convierte una categoría móvil, surgida de la combinación de la concordancia de la historia y la discordancia que imponen los acontecimientos, y por lo tanto, constantemente abierta a su reelaboración. En segundo lugar, la disociación de esta categoría respecto de cualquier sustrato objetivo, permite aplicarlo tanto a los individuos como a grupos. Por último, al no existir tampoco una determinación acerca de quién o quiénes son los que llevan a cabo el proceso de constituir una narración de este tipo, Ricoeur está posibilitando la intervención de terceros en su elaboración. De este modo, nuestra identidad sería constituida, entre otras cosas, a partir de las historias contadas por los demás, pues hay muchísimos aspectos de nuestras vidas que no están a nuestro alcance poder narrarlas, como es el caso de nuestro nacimiento y primeros años de vida. Esto significa, correlativamente, que también la identidad de los otros está en parte constituida por historias contadas por nosotros.

Teniendo presentes estos aportes de Ricoeurse, se está en condiciones de caracterizar en qué consiste este segundo nivel de integración. A grandes rasgos el intercambio de memorias apunta a identificarse con la historia de nuestros semejantes, para lograr a partir de allí reconfigurar nuestro pasado y el de los demás. No se trata de revivir lo que vivieron los otros, como lo había hecho Dilthey, ni un proceso empático a lo Husserl, sino en un intercambio de memorias en el nivel narrativo. En la medida en que la identidad no es rígida, es posible revisar los relatos que cada grupo tiene de sí mismo y reestructurarlo a partir de los relatos de los grupos vecinos. Aquí aparece el primero de los aspectos terapéuticos que retomaremos en el siguiente punto ligado a la conmemoración de los acontecimientos fundacionales bases para la identificación del imaginario colectivo. Según el filósofo francés, "lo que realmente impide a las culturas poder ser contadas de manera diferente es la influencia ejercida sobre la memoria colectiva por lo que denominamos los 'acontecimientos fundacionales', cuya conmemoración y celebración tienden a congelar la historia de cada grupo cultural en una identidad que no es sólo inmutable pero también deliberada y sistemáticamente incomuni- 
cable." (Ricoeur, 1995, p. 7). Sin embargo, en lugar de proponer abandonar estas marcas históricas, el filósofo propone una lectura plural de ella.

El tercer nivel de integración apunta al perdón como forma específica de revisión del pasado y de las identidades narrativas ligada a acontecimientos traumáticos. Este fenómeno se asocia con lo que se ha dado en llamar el 'terror de la historia'. Ricoeur coincide de alguna manera con Benjamin al sostener que el intercambio de memorias no se puede estar guiado por el tradicional presupuesto de gloria de la historia ("la historia es de los vencedores"), sino de la perspectiva del sufrimiento ("la historia es de los vencidos"). Es preciso empezar por el sufrimiento de los otros, imaginándolo antes de reexaminar los de uno.

Hay un momento, empero, en que el eje de atención se desplaza de las víctimas hacia los victimarios. En efecto, siguiendo a Todorov, Ricoeur propone abandonar la memoria literal que se queda fijada en el pasado y, en su lugar, propone una memoria ejemplar, que busca poner fin a los traumatismos apoyándose en el futuro. ${ }^{6}$ En lugar de sacralizar a las víctimas y hacer un culto del pasado, se trata es de reintegrar a los victimarios a la sociedad, y no de vengarlos eternamente por el daño que infringieron. No se trata de olvidar el pasado, sino de desacoplar al agente de su acción. Esta disociación permite perdonar al individuo que la llevó a cabo una acción particular, sin condonar a la acción misma. Como lo explica el filósofo, "me acerco, una vez más, al argumento de Derrida: separar al culpable de su acto, en otras palabras, perdonar al culpable sin dejar de condenar su acción, sería perdonar a un sujeto totalmente distinto que cometió el acto." (Ricoeur, 2000, 628). Ricoeur destaca que la justicia y el perdón funcionan a niveles diferentes y con lógicas diferentes. Mientras que la justicia se basa en la reciprocidad, en el perdón existe una asimetría hiperbólica. Sin embargo, el perdón no busca reemplazar a la justicia, ni abolir la deuda. Se trata, por el contrario, de levantar la barrera de la culpa que paraliza las relaciones entre individuos que están repitiendo y sufriendo su propia historia para abrirnos hacia el futuro.

\section{III}

En su capítulo cuarto Uggla plantea que la globalización podría llevar a nivel político-cultural a una guerra de memorias. Dentro de este contexto, este autor sostiene que "hoy, me parece, que uno de los mayores retos es transformar la noción de un amenazador choque de las civilizaciones (S. Huntington) en un conflicto de las interpretaciones (P. Ricoeur), que significa un modelo pacífico de resolución de conflicto." (Uggla, 2010, p. 84). Con todo, quisiéramos mostrar de qué modo la falta de las remisiones

${ }^{6}$ Cf. Ricoeur, 2000 pp. 104-105. 
Consideraciones en torno a la globalización en el marco de una hermenéutica rico euriana

a la obra de Freud y del modelo de integración arriba señalado lleva a que este intérprete pierda de vista uno de los aportes más importantes de la hermenéutica ricoeuriana, a saber: su dimensión terapéutica.

Uggla toma la tipología de usos y abusos de la memoria desarrollada en La memoria, la historia, el olvido como marco para un análisis comparativo entre la memoria finlandesa y la sueca. En su análisis, destaca tres elementos de las representaciones mnémicas de estos pueblos. En primer lugar, a pesar de que la independencia finlandesa fue el 6 de diciembre de 1917, su conmemoración siempre remite a las Guerras de Invierno y la Continuación contra la Unión Soviética acontecidas dos décadas más tarde. La explicación de este desplazamiento se debería a la cercanía entre la fecha de la independencia y una 'traumática' guerra civil acontecida al año siguiente de dicha fecha. Este hecho lleva a que el autor ponga de manifiesto de la tipología de Ricoeur toma la forma de un proceso, en el que los tres momentos están relacionados pero no ordenados jerárquicamente. "la memoria impedida (en un nivel patológico-terapéutico) del gran número de muertos [...] estos acontecimientos todavía dividen a los finlandeses entre amigos y enemigos. [...] Esta memoria manipulada (en un nivel práctico) es combinada con la memoria obligada (en un nivel ético político) de la experiencia compartida de un acontecimiento verdaderamente unificador cuando Finlandia peleó con un enemigo externo común, como en la Guerra de Invierno." (Uggla, 2010, p. 98). En segundo lugar, también propone la tesis de la memoria impedida para explicar la ausencia de celebraciones durante las conmemoraciones de la fecha patria sueca. Esta actitud, así como su internacionalismo se deberían fundamentalmente a las derrotas militares y pérdidas territoriales durante los últimos doscientos años, y a sus simpatías con el régimen Nacional Socialista, durante la Segunda Guerra Mundial. Por último, Uggla compara el comportamiento dentro de la Unión Europea de estos dos modelos de identidad, uno fuerte, basado en el sentimiento de heroísmo romántico decimonónico que proporciona todo triunfo en guerras independentistas y, por el otro, uno débil debido a las vergüenzas padecidas durante los últimos doscientos años. De esta comparación, concluye que "las experiencias de más de una década de membrecía en la Unión Europea pueden apoya la idea que Finlandia parece está mejor preparada para actuar más allá del marco nacional." (Uggla, 2010, 107).

Considero limitado el análisis de los primeros dos aspectos aquí resumidos pues asocian ciertas actitudes colectivas con la taxonomía de usos y abusos de la memoria y sobre ella se permiten hacer una consideración teórica acerca de la posibilidad de tomar esta taxonomía como un proceso. Con ello, empero, se pasa por alto los aspectos más ricos de la hermenéutica ricoeuriana. En efecto, el filósofo francés coincide con Michel de Certeau en que la historia no sólo establece criterios para comprender las diferencias y continuidades entre el presente y el pasado y explica el pasado, sino que también debe elaborar maneras de pensar y superar la violencia de los conflictos 
de la historia. ${ }^{7}$ En su análisis de la memoria impedida, plantea la posibilidad de aplicar los resultados de la metapsicología freudiana, especialmente los referidos al duelo y la melancolía a nivel colectivo. Parte del desarrollo ontológico de esta obra, busca proporcionar los fundamentos necesarios para que esta aplicación sea posible y que el historiador se pueda constituir en una suerte de analista colectivo. En el hipotético diálogo entre Certeau y Heidegger, el filósofo reemplaza lo que denomina la vía corta heideggeriana, que establece un vínculo directo entre el poder-ser y la muerte, por otra larga, que abra al Dasein a la exterioridad y la factualidad, restituyendo así la densidad ontológica del se muere impropio. ${ }^{8}$ Esto significa que, de manera análoga a lo que sucede en el Dasein, a nivel colectivo habría una primacía del advenir de manera análoga respecto del sido y el presente.

Si el autor hubiera tomado en cuenta estos aspectos, se habría detenido en el tipo de tarea que debería llevar a cabo la historia para lograr "los cambios de actitud en el ethos de los individuos, grupos y gentes," ${ }^{9}$ más que establecer cuál de los dos modos de asumir la identidad nacional está mejor preparada mantenerse dentro de un bloque supranacional. En este sentido, los aportes del modelo ricoeuriano de integración, especialmente el perdón y el intercambio de memorias, permitirían desbloquear a ambas memorias impedidas. En efecto, el perdón permitiría, por un lado, poder dejar de lado las diferencias internas que separan a los finlandeses entre sí, posibilitando que sus festejos de independentistas puedan volver a remitirse a los sucesos de 1917. Por la otra, el perdón de los suecos respecto de su actuación en la Segunda Guerra haría que los suecos superen su desazón nacional que impida que desdibujen su identidad en un vago internacionalismo.

\section{IV}

Una vez llegados a este punto, es preciso que destaquemos un punto que va más allá de las coincidencias entre Uggla y Ricoeur y, podríamos decir, las posibilita: ambos autores abordan la problemática de la globalización desde la hermenéutica. Históricamente la hermenéutica ha estado ligada con la historia intelectual, conceptual o de las ideas, en tanto que el problema de la globalización está vinculado a la historia social. La pregunta que cabe hacerse aquí es cómo es posible que un autor identificado con la hermenéutica, como es el caso de Ricoeur, pueda llegar a afirmar que “...la historia social no es un sector entre otros, sino el punto de vista bajo el cual la historia elige su campo, el de las ciencias sociales." (Ricoeur, 2000, 232), y que lleve sus análisis históricos en esta dirección. Quisiéramos detenernos en dos respuestas complementarias. Mientras que

${ }^{7}$ Cf. de Certeau, 1995, p. 79.

${ }^{8}$ Cf. Ricoeur, 2000, p. 475.

${ }^{9}$ Cf. Ricoeur, 2010, p. 5. 
Consideraciones en torno a la globalización en el marco de una hermenéutica rico euriana

la primera proviene de la historia del seno mismo de la historia, la segunda se detiene a señalar aquellos aspectos presentes en La memoria, la historia, el olvido que lo posibilitan.

Durante mucho tiempo la historia intelectual/cultural y la historia social se han considerado como ámbitos de investigación separados entre sí. La primera analizaba el desarrollo de las ideas de diferentes autores y su mayor proceso de contextualización consistía en relacionarlo con autores contemporáneos, pretéritos y subsiguientes. La segunda, por su parte, se centraba en los procesos sociales, focalizándose en cuestiones como las reveliones internas, guerras y consideraciones en torno a la historia. Varios investigadores consideran que la frontera entre ambas fue rota a partir de la recepción realizada por la historia del giro lingüístico, durante la década de los setenta. Un autor cercano a la historia intelectual, como es el caso de François Dosse, considera que ha este giro ha modificado el panorama histórico en dos aspectos. "La otra gran mutación tiene que ver con los efectos del linguistic turn, que ha dado nueva centralidad al 'mundo del texto' y una mayor atención tanto a la operación de la escritura como a su recepción por múltiples espacios de lectura, en función del lugar y del momento de su apropiación." (Dosse, 2007, p. 128). En este proceso, Dosse destaca la influencia que han tenido autores como de Certeau, Foucault y Chartier.

Desde la historia social, Geoff Eley también vincula este proceso con el giro lingüístico. Este autor identifica tres motivos que conducen a lo que él denomina "la crisis de la historia social": 1. La irrupción de la teoría de género y su propuesta de una lectura del pasado enfrentada con los modelos y categorías que existían dentro de la historia social, 2. La lectura histórica de Foucault, especialmente su concepto de discurso como medio de teorizar a la vez las reglas internas, las regularidades de los campos del saber y las estructuras generales de las ideas y de los presupuestos que delimitan lo que puede y no puede ser dicho y pensado, 3. La historia de las mentalidades como vía para acceder a la cultura popular del pasado, frente a la alternativa elitista de la historia cultural. (Eley, 1992, p. 172).

No son novedosos los cruces que realizó Ricoeur entre la hermenéutica y el mundo de la acción, como lo ponen de manifiesto su uso en Tiempo y relato de la teoría de la acción como vía para establecer continuidades entre la configuración narrativa y la acción humana. Incluso, los cruces que hace Roger Chartier entre prácticas y representaciones están inspirados en las categorías ricoeurianas de 'mundo del texto' y 'mundo del lector'. ${ }^{10}$ Sin embargo, a diferencia de lo que sucede en las obras anteriores, donde es el propio Ricoeur, el que propone diversos vínculos entre el mundo de la acción y el del texto, en La memoria, la historia, el olvido recoge los desarrollos realizados por los historiadores que él mismo inspiró en lo referente a la representación y los articula con las demás dimensiones de la representación por él tratadas. En efecto, su

${ }^{10}$ Cf. Dosse, 2007, p. 135, 
análisis de la fase explicativa/comprensiva de la operación histórica es un análisis del concepto de representación tal como es desarrollado por la cuarta generación de los Annales. En primer lugar, cuando se plantea este concepto se lo hace con el objeto de precisar algo más el concepto de mentalidad, es decir, se acerca al primero de los motivos señalados por Eley, que ya se había vuelto demasiado ambiguo para seguir siendo utilizado. El recorrido que hace el filósofo por los 'maestros del rigor', Foucault - es decir, el segundo de los causantes de la crisis de la historia social -, de Certeau y Elias, y su análisis de la variación de escala, le permite poner de manifiesto la articulación que existe, por una parte, entre representaciones y prácticas sociales $\mathrm{y}$, por la otra, entre individuo, grupo y estado. ${ }^{11}$

Con otras palabras, así como en la época de Tiempo y Narración Ricoeur recurrió a la teoría de la acción como vía para establecer las analogías entre la configuración narrativa y la acción humana, en La historia, la memoria, el olvido este rol será cumplido por la 'representación'. Como lo sostiene el propio filósofo francés, "habría una relación mimética entre la representación-operación, en tanto que momento del hacer la historia, y la representación-objeto, en tanto que momento del hacer historia." (Ricoeur, 2000, p. 295)

A lo largo de estas páginas nos hemos detenido en la obra de Paul Ricoeur porque coincidimos con Bengt Kristensson Uggla en que ésta aporta elementos para resolver algunos problemas que surgen del proceso de la globalización. Pese a esta coincidencia general, también nos detuvimos para poner de manifiesto las diferencias que nos separaban de este intérprete. En primer lugar, rechazamos su propuesta de considerar a la hermenéutica meramente como un tipo de racionalidad alternativo al relativismo horizontal postmoderno y al intento del pensamiento vertical de jerarquías fijas de verdad pues, sosteníamos. En contraposición consideramos que ésta proporciona de contenidos concretos para abordar problemáticas ligadas a la globalización. En efecto, el ethos de la traducción, el intercambio de memorias y el perdón proporcionarían un ámbito para cerrar diferencias y asimetrías entre lo que hemos denominado el proyecto globalizador desde arriba y otro realizado desde abajo.

En segundo lugar, nos detuvimos a la aplicación que hacía Uggla de los análisis ricoeurianos de la memoria a los casos de Finlandia y Suecia, observábamos que en ellos se pasaba por alto un elemento terapéutico presente en Ricoeur, que proporcionaría el fundamento y los elementos para resolver los traumas colectivos que afectan a

${ }^{11}$ Cf. Ricoeur, 2000, 302-373 
Consideraciones en torno a la globalización en el marco de una hermenéutica rico euriana

nivel de la memoria colectiva. En este sentido, consideramos que antes que discutir cuál representación mnémica es más apta para afrontar un mundo globalizado, es más importante establecer estrategias terapéuticas que pueden ser llevadas a cabo a nivel colectivo para desbloquear estas memorias impedidas.

Finalmente, quisimos resaltar un punto que suele ser pasado por alto en los análisis de la obra de Ricoeur, y es su desplazamiento de temáticas ligadas con la lectura y la estética de la recepción hacia problemáticas sociales. En este sentido, hemos señalado la importancia que ha tenido la recepción del giro lingüístico en historia a fin de que la hermenéutica pudiera abordar problemas tradicionalmente ligados con la historia social, como es la globalización.

\section{Bibliografía:}

De CERTEAU, Michel, Historia y Psicoanálisis entre ciencia y ficción, Universidad Iberoamericana, México, 1995.

DOSSE, François, La marcha de las ideas. Historia de los intelectuales, historia intelectual, Universitat de València, Valencia, 2007.

ELEY, G., “De l'histoire sociale au 'tournant linguistique' dan l'historiographie angloaméricaine des années 1980, en Genèses 7 (163-193), 1992.

FRIEDMAN, Thomas, The Lexus and the Olive Tree, Farrar Strauss Giroux, New York, 1999.

HARDT, Michael, NEGRI, Antonio, Empire, Harvard University Press, Cambridge, 2000.

KELLNER, Douglas, “Theorizing Globalization” en Sociological Theory 20:3, Noviembre 2002 (285-305)

KRISTENSSON UGGLA, Bengt Ricoeur, Hermeneutics and Globalization, Continuum International Publishing Group, New York. 2010.

RICOEUR, Paul. "Reflections on a new ethos for Europe" en Philosophy and Social Criticism, vol. 21 5/6 1995 (3-13) - . La mémoire, l'histoire, l'oublie. Paris: Seuil, 2000

STIGLITZ, Joseph E., Globalization and its Discontents, Norton, New York, 2002. 